\title{
Planeamiento territorial sostenible: un reto para el futuro de nuestras sociedades; criterios aplicados
}

\author{
Sustainable spatial planning: a challenge \\ for the future of our societies; applied criteria
}

Adrián Ferrandis Martínez Joan Noguera Tur

\begin{abstract}
Resumen
En buena parte de los 17 objetivos de desarrollo sostenible marcados como metas de la humanidad por la ONU se deja entrever la sostenibilidad. Debido al modelo socioproductivo dominante, el único modo de avanzar hacia territorios más sostenibles que permitan alcanzar y mantener el bienestar de la población mundial, es tener presente la necesidad de planificar adecuadamente el desarrollo territorial. Este trabajo reflexiona sobre esta necesidad y avanza en la definición de los principales criterios para alcanzar la sostenibilidad territorial en las escalas regionales y locales.
\end{abstract}

Palabras clave: criterios de sostenibilidad; planeamiento territorial; desarrollo sostenible; sociedades sostenibles y planificación urbana.

\begin{abstract}
In a large part of the 17 sustainable development objectives set as goals for humanity by the UN, sustainability can be glimpsed. As a result of the dominant socio-productive model, the only way to head towards more sustainable territories that allow achieving and maintaining the well-being of the world's population is to bear in mind the need to properly plan territorial development. This work reflects on this need and takes a step forward in the definition of the main criteria to achieve territorial sustainability at regional and local scales.
\end{abstract}

Keywords: sustainability criteria; spatial planning; sustainable development; sustainable societies and urban planning. 


\section{Introducción}

La mejora del grado de sostenibilidad de los territorios y en especial de los espacios urbanos y metropolitanos, es uno de los principales retos para el futuro de las sociedades actuales. De la medida en la que esta mejora resulte una realidad plausible, dependerá el conseguir salvaguardar el equilibrio del sistema ecológico de nuestro planeta, y con él, las condiciones de vida que hoy conocemos (Rueda et al., 2012).

Los principios establecidos en la teoría del Desarrollo Sostenible (DS), representan el modelo de desarrollo de referencia para conseguir estas mejoras sustanciales. A pesar de esto, la importante carga de ambigüedad que rodea al concepto del DS, requiere avanzar hacia planteamientos más prácticos que permitan caminar hacia aquello que ha venido a denominarse como sostenibilidad fuerte (Naredo, 1996). Es decir, concretar en mayor medida los criterios de acción necesarios para aplicar políticas de desarrollo territorial que resulten lo más sostenibles posibles, desde un punto de vista global en el que se integren los ámbitos sociales, ambientales y económicos.

Este artículo tiene como objetivo resaltar la importancia de la Planificación Territorial como instrumento fundamental para la aplicación del DS, y mejorar el grado de sostenibilidad de los territorios, con una relevancia especial en las escalas regionales y locales. A continuación se presentan diferentes apartados que irán desgranando cada uno de los conceptos clave para su interpretación, y en los que, finalmente, a partir de una minuciosa revisión bibliográfica, se definen los criterios de sostenibilidad fundamentales para su implementación práctica.

\section{El reto del desarrollo sostenible}

La consecución de aquello ha venido a denominarse como DS, es presentado por numerosos autores como uno de los retos más importantes para salvar la Tierra de los males que la aquejan. Males causados, y sobre todo acelerados, por la acción antrópica sobre el Planeta, y en especial sobre su sistema natural o ecológico (Ramonet, 2003). La pérdida de Biodiversidad, las alteraciones climáticas, la contaminación atmosférica, la intensificación de los procesos de desertización o el aumento de la pobreza humana, son algunos de esos males que lo afectan y pueden provocar su transformación, alterando por tanto las actuales condiciones de vida.

El término sostenibilidad tiene que ver con la garantía de continuidad en el tiempo de las características estructurales y funcionales de los valores y de los atributos de aquello a lo que hace referencia, ya sea un sistema, un recurso o una actividad (Gómez Orea, 2007). Etimológicamente la palabra contiene connotaciones interesantes e importantes, pues se puede referir al hecho de apoyar un estado deseado, o por el contrario, a soportar un estado no deseado. En términos generales, cuando se hace referencia a algo que es sostenible, se quiere identificar una acción o proceso que es capaz de ser mantenido o defendido. Al mismo tiempo, si se utiliza como un elemento más activo, sugiere una disposición hacia algo, que 
trasmite un mensaje prescriptivo de que algo hay que hacer, y que de hecho puede hacerse (Redclift, 2000).

Por su parte, el término desarrollo está vinculado al hecho de acrecentar o dar incremento a algo de orden físico, intelectual o moral, así como también al concepto de progresar o crecer económica, social o culturalmente (RAE, 2014).

En el año 1987, como resultado de los trabajos realizados por la Comisión Mundial del Medio Ambiente y del Desarrollo, presidida por la entonces ministra de medio ambiente noruega Gro Harlem Brundtland, se elaboró el informe denominado Nuestro Futuro Común, más conocido como Informe Brundtland. Sin duda alguna, este es el documento de referencia a la hora de hablar y profundizar en el conocimiento y definición del DS, y el que le otorgó su acuñación oficial. En una de las partes del informe se desmenuza el concepto de DS, y se indican todas y cada una de las connotaciones y campos a los que afecta, definiéndolo en suma de la siguiente forma:

El DS es el proceso de cambio en el cual la explotación de los recursos, la orientación de la evolución tecnológica y la modificación de las instituciones, están acordes y acrecientan el potencial actual y futuro para satisfacer las necesidades y aspiraciones humanas. (Informe Brundtland, 1987, p. 19)

Otra definición más sintética, es aquella que lo define como:

La posibilidad de satisfacer las necesidades del presente sin comprometer la capacidad de las futuras generaciones para satisfacer las suyas. (Informe Brundtland, 1987, p. 21)
Al final del proceso, las diferentes cuestiones acordadas se plasmaron en una serie de principios legales generales en pro de la protección del medio ambiente y el DS. Desde el punto de vista del interés que suscitan por la temática de este artículo, se considera importante destacar los siguientes:

- Los derechos de todos los seres humanos a tener un medio ambiente adecuado para su salud y bienestar.

- La conservación y utilización del medio ambiente y los recursos naturales para el beneficio de las generaciones presentes y futuras.

- El mantenimiento de los ecosistemas y los procesos ecológicos indispensables para el funcionamiento de la biosfera y la conservación de la diversidad biológica.

- La incorporación del concepto de "óptimo rendimiento sostenible" en la utilización de los recursos naturales vivos y de los ecosistemas.

- El requerimiento de evaluaciones previas de las actividades que se propongan y puedan afectar considerablemente el medio ambiente o a la utilización de recursos naturales.

- La pretensión de asegurar la conservación como parte integrante de la planificación y ejecución de las actividades de desarrollo.

- La proporción de asistencia especial a los países en desarrollo en apoyo a la protección del medio ambiente y el DS.

- La utilización de los recursos naturales transfronterizos de manera razonable y equitativa.

- El requerimiento de una evaluación ambiental de las actividades proyectadas que pudieran tener importantes efectos transfronterizos. 
En definitiva, el informe asentó las bases para la definición de una teoría alternativa a las teorías económicas predominantes, enmarcadas en el crecimiento ilimitado (Goodland, 1997). El consumo descontrolado e intensivo de los recursos naturales había provocado una degradación de los activos naturales, por lo que, mientras el imparable crecimiento económico aumentaba, el sistema natural, se empobrecía. Además, junto a estas actividades de consumo de recursos de forma ilimitada, se le sumaba otra, vinculada a la generación de residuos, como resultado de las actividades productivas de transformación, causantes de la contaminación del aire urbano, las aguas, así como el efecto invernadero y la disminución del ozono de las capas estratosféricas.
Como principio general, el concepto del DS se clarifica en esencia mediante una triple dimensionalidad; la económica, la social y la medioambiental o ecológica (Douglas Muschett, 1999; Lee Cambell y Heck, 1999; Gómez Orea 2007). El espacio ocupado por la sostenibilidad, dentro de esa triple dimensión, es justo la zona de contacto en la que se superponen las tres a la vez (observar la Figura 1). Si las actividades 0 líneas de crecimiento quedaran fuera de ese espacio de superposición, pasarían a situarse en las zonas del desarrollo no sostenible. Se puede argumentar que la zona de contacto representa el punto de equilibrio en el que se puede combinar el crecimiento económico, el progreso social y el respeto al medio ambiente.

Figura 1 - Intersección de los elementos clave que engloba el DS

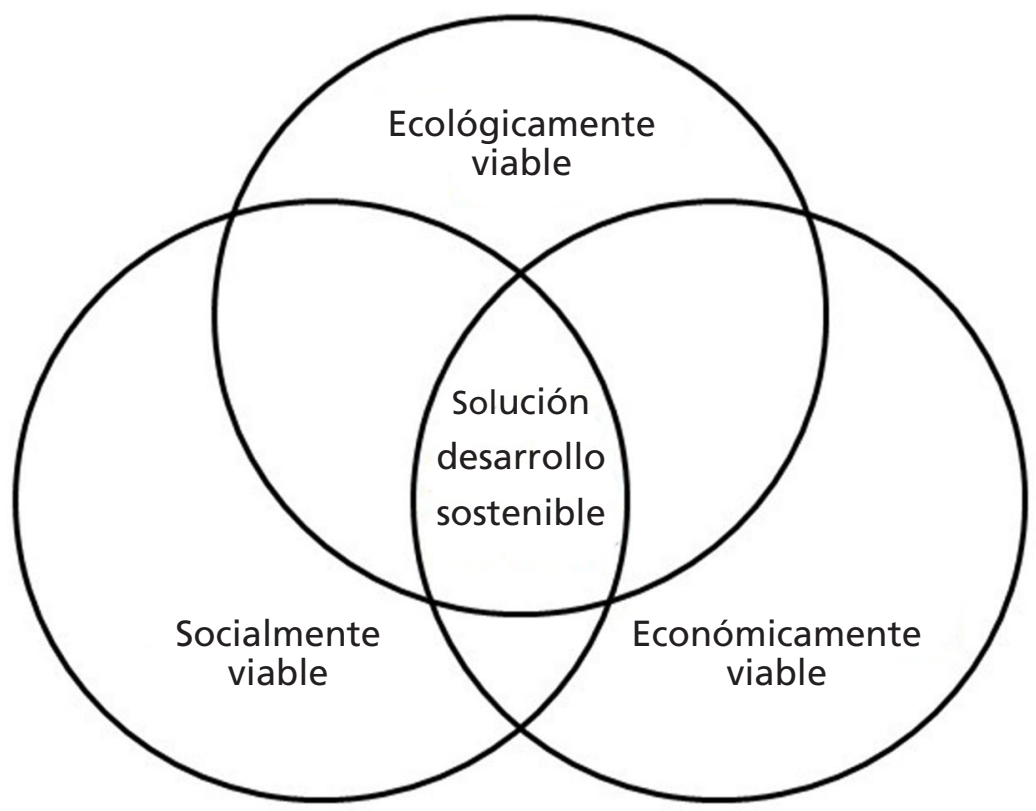

Fuente: Elaboración propia, a partir de Lee Cambell y Heck (1999). 
Esa triple identificación conduce a interpretar el DS en términos de calidad de vida y, valorada dentro del plano individual, se puede traducir en tres componentes: el nivel de renta, las condiciones de vida y de trabajo y la calidad ambiental de cada individuo, junto a los que forman la sociedad (Gómez Orea, 2007). En definitiva, el concepto de DS se inscribe dentro de la idea optimista que considera que es compatible el desarrollo económico y la conservación del medio ambiente, o lo que es lo mismo, del medio/recurso natural sobre el que se asientan las sociedades.

A partir de estas aproximaciones iniciales, el concepto del DS ha sido evolucionado mediante la concreción de sus principios. Como se puede interpretar en la figura que se muestra a continuación (Figura 2), tomando como referencia el análisis elaborado por Ferrandis (2016), a partir de los principales argumentos defendidos por distintos autores de referencia, el DS queda definido desde una perspectiva integral. Destacan principios y objetivos como los relacionados con la necesidad de instaurar procesos productivos que primen la eficiencia, la conservación de los recursos, el reciclaje o la necesidad de invertir en sectores relacionados con el medio ambiente (Goodland, 1997; Von Droste y Dogsé, 1997; Shen, 1999; Lee
Cambell y Heck, 1999). Reducir la generación de residuos y prevenir posibles proceso de contaminación mediante su tratamiento adecuado (Douglas Muschett, 1999; y Shen, 1999). La necesidad de provocar cambios sociales y culturales, especialmente en la forma de interpretar la relación del hombre con el medio natural que le rodea (Douglas Muschett, 1999; y Vilches y Gil, 2003). Esto último se podrá conseguir incidiendo en los procesos educativos. Controlar el hiperconsumo e intentar desconectarlo de su asociación directa con la mejora de la calidad de vida (Goodland, 1997; Vilches y Gil, 2003). Fomentar el desarrollo en base a elementos cualitativos, como puede ser todo lo que tenga que ver con las energías renovables, las comunicaciones y otros sectores estratégicos (Von Droste y Dogsé, 1997; Shen, 1999; Lee Cambell y Heck, 1999; Vilches y Gil, 2003). Instaurar la universalización de los Derechos Humanos y la democratización institucional a nivel planetario (Lee Cambell y Heck, 1999; Vilches y Gil, 2003). 0 reducir el hambre, la pobreza y la exclusión social, mediante la redistribución de los recursos en base a elementos de índole temporal (solidaridad intergeneracional) y espacial (Norte/Sur) (Redclift, 2000; Vilches y Gil, 2003). 
Figura 2 - Tabla de síntesis de los principios y objetivos de la evolución del concepto del D.S.

\begin{tabular}{|c|c|c|c|c|c|c|c|c|}
\hline \multirow[b]{2}{*}{$\begin{array}{l}\text { Principios y objetivos fundamentales de la } \\
\text { evolución conceptual del D.S. }\end{array}$} & \multicolumn{8}{|c|}{ Autores y año de publicación } \\
\hline & $\begin{array}{c}\text { Goodland } \\
\text { (1997) }\end{array}$ & \begin{tabular}{|l|} 
B. Von \\
Droste y \\
P. Dogsé \\
$(1997)$ \\
\end{tabular} & $\begin{array}{l}\text { Douglas } \\
\text { Muschett } \\
\text { (1999) }\end{array}$ & $\begin{array}{l}\text { Shen } \\
(1999)\end{array}$ & $\begin{array}{l}\text { Lee } \\
\text { Cambell } \\
\text { y Heck } \\
\text { (1999) }\end{array}$ & $\begin{array}{l}\text { Redclift } \\
(2000)\end{array}$ & $\begin{array}{l}\text { Vilches } \\
\text { y Gil } \\
\text { (2003) }\end{array}$ & $\begin{array}{l}\text { Gómez } \\
\text { Orea } \\
\text { (2007) }\end{array}$ \\
\hline $\begin{array}{l}\text { Producir más con menos (conservación, } \\
\text { eficiencia, reciclaje y inversión en sectores } \\
\text { meioambientales) }\end{array}$ & $x$ & $\mathrm{x}$ & & & & & & \\
\hline Reducir la explosión demográfica & $x$ & & $x$ & & & & $x$ & \\
\hline Reducir el sobreconsumo o hiperconsumo & $x$ & & & & & & $x$ & \\
\hline $\begin{array}{l}\text { Fomentar el desarrollo en base a elementos } \\
\text { cualitativos (nuevas tecnologías) }\end{array}$ & $x$ & $\mathrm{x}$ & $\mathrm{x}$ & $\mathrm{x}$ & & & $x$ & \\
\hline $\begin{array}{l}\text { Inversiones en investigación y produción } \\
\text { ámbito biotecnología (energias renovables, } \\
\text { comunicaciones, etc.) }\end{array}$ & & $\mathrm{x}$ & & $\mathrm{x}$ & $\mathrm{x}$ & & $\mathrm{x}$ & \\
\hline $\begin{array}{l}\text { Redución resíduos y prevención } \\
\text { de la contaminación }\end{array}$ & & & $x$ & $x$ & & & & \\
\hline $\begin{array}{l}\text { Formación (educación) y cambios sociales } \\
\text { y culturales }\end{array}$ & & & $x$ & & & & $x$ & \\
\hline $\begin{array}{l}\text { Reducir el hambre, la pobreza y la exclusión } \\
\text { social }\end{array}$ & & & & & $\mathrm{x}$ & & $x$ & \\
\hline $\begin{array}{l}\text { Universalización de los DDHH y } \\
\text { democratización institucional a nivel planetario }\end{array}$ & & & & & $\mathrm{x}$ & & $x$ & \\
\hline $\begin{array}{l}\text { Redistribución de los recursos en base } \\
\text { a elementos como el tiempo (ahora/luego: } \\
\text { igualdad intergeneracional y el espacio } \\
\text { Norte/Sur) }\end{array}$ & & & & & & $x$ & $x$ & \\
\hline $\begin{array}{l}\text { Triple dimensionalidad del D.S.: económica, } \\
\text { social y ambiental }\end{array}$ & & & $\mathrm{x}$ & & $\mathrm{x}$ & & & $x$ \\
\hline
\end{tabular}

Fuente: Ferrandis (2016).

En los años posteriores a los indicados en la figura de síntesis de los principios y objetivos del DS, se puede destacar que pocos autores han aportado modificaciones sustanciales a sus planteamientos, por lo que han centrado sus críticas y valoraciones en la forma en la que se está intentando aplicar el DS y, especialmente, en el enfoque exageradamente desarrollistas de los principales agentes implicados (Besset, 2005; Latouche, 2008; Urteaga, 2011). Esta situación ha derivado en la propuesta de teorías o planteamientos alternativos al propio
DS (Decrecimiento, Ecodesarrollo, etc.), pero que como ya se ha indicado, distan poco de sus principios fundamentales y centran sus propuestas de cambio en elementos clave, como puede ser la necesidad de implementar un cambio cultural en nuestras forma de vida, en la que la felicidad y el bienestar no dependa única y exclusivamente de la posesión de determinados bienes materiales (Latouche, 2008), o en la necesidad de sobreponer los intereses del bien común (del conjunto de ciudadanos del Planeta) sobre los 
interés particulares de los grandes poderes económicos del Mundo y del capitalismo en general (entendido este como aquella parte del engranaje del sistema capitalista que sólo prima la vertiente económica, menospreciando la social y la ambiental). En definitiva, bajo estos "nuevos" 1 enfoques, lo que se intenta es hacer valer la incapacidad de la clase política actual para liderar ese cambio tan necesario, que sucumbe bajo el interés de unos pocos, los acumuladores de capital.

\section{La Planificación Territorial; un instrumento político de acción necesario para alcanzar el desarrollo sostenible}

Durante el siglo XIX, como consecuencia de la revolución industrial y del crecimiento demográfico, en los países occidentales, se produjeron unas décadas de convulsión urbana, que pusieron de manifiesto la necesidad de ordenar el crecimiento de las ciudades mediante políticas, normativas, instrumentos y competencias públicas (Cruz Villalón, 2006). Evolucionado dicho proceso urbano, ya a principios del siglo $\mathrm{XX}$, se constató, que ni los problemas urbanísticos, ni los derivados de la expansión urbana, podían ser resueltos, ni abordados, exclusivamente desde la escala local. Correspondería entonces a lo que ha venido a denominarse Ordenación del Territorio (OT), la responsabilidad de afrontar en la escala supralocal, la resolución de los problemas urbanísticos y de cualquiera otros que afectasen a los territorios y a las sociedades que se asientan en ellos.

Sin embargo, un siglo después, la OT no se encuentra completamente consolidada, y todavía se discute el contenido específico de la planificación territorial, cuáles son los instrumentos propios de la OT y la escala o ámbito de aplicación (Cruz Villalón, 2006).

La Planificación Territorial (PT), entendida como un instrumento de acción de la OT, se justifica porque la conflictividad inherente a cualquier sistema territorial, dejado a su evolución espontánea, se resuelve en beneficio del interés privado del más fuerte, con objetivos a corto plazo, lo que conduce a un sistema territorial insatisfecho. Este hecho es el que justifica la intervención planificada sobre el denominado sistema (Gómez Orea, 2007).

El territorio es el resultado de la construcción sociológica que los humanos han levantado sobre la matriz biofísica preexistente, creando un espacio de artificialidades oportunas, en ocasiones, y no tanto en otras (Folch, 2003). Este territorio debe ser entendido como algo más que un mero escenario, ya que cuenta con una naturaleza sistémica y compleja que se resiste a toda percepción reduccionista. El territorio está compuesto por la ocurrencia de elementos y fenómenos interrelacionados que configuran su realidad, es decir, la realidad territorial de cada una de las sociedades.

Otro elemento importante, que también se debe considerar, es que el territorio tiene un carácter limitado, y por tanto es un bien escaso y finito, por lo que resulta de gran relevancia su adecuada planificación y ordenación, de tal forma que se maximicen los efectos 
potenciales de su buena gestión (Pujadas y Font, 1998; Farinós, 2006; Esteban, 2006; Gómez Orea, 2007).

La PT se debe aplicar a todos los niveles territoriales: nacional, regional y local, mediante un conjunto de planes, cuyas determinaciones tienen que corresponder con las estructuras territoriales atribuibles a cada nivel. Estas estructuras se desarrollan en cascada, según un proceso de arriba abajo, en el que el nivel superior, y ámbito más extenso, se adopta como referencia para los niveles inferiores (Pujadas y Font, 1998; Gómez Orea, 2007; Noguera, 2009). Tal desarrollo en cascada no implica la imposición de los niveles superiores sobre los inferiores, sino una correlación entre las estructuras territoriales asociadas a cada nivel y el tipo de plan correspondiente. De acuerdo a ello, toda población participa en la elaboración de todo plan.

El objetivo final de la PT es el desarrollo global de los sistemas territoriales a los que se aplica, entendido éste en términos de calidad de vida. El desarrollo integral implica equilibrio, integración, funcionalidad, uso racional de los recursos, calidad ambiental, calidad de la gestión pública y coordinación administrativa.

Cuando se habla de equilibrio territorial, lo que se pretende es prevenir y corregir los desequilibrios territoriales, ya que el equilibrio entre las diferentes unidades territoriales es garantía de progreso y estabilidad. Por tanto, la PT debe controlar el crecimiento de las regiones demasiado dinámicas y estimular el de las retrasadas o las que entren en decadencia (Carta Europea de Ordenación del Territorio, 1983).

Por su parte la idea de la integración (territorial y sectorial, hacia arriba y hacia abajo), implica una especie de justicia social, que concilia los intereses conflictivos de los diferentes agentes socioeconómicos y hace prevalecer el interés de la comunidad sobre los intereses privados. A su vez el elemento de funcionalidad, vinculado con la optimización de las relaciones entre las actividades a través de los flujos de relación que se producen entre ellas, implica una organización espacial, un control del uso del suelo, una accesibilidad de la población a la explotación de los recursos territoriales, a los lugares de trabajo y a los equipamientos y servicios públicos (Gómez Orea, 2001).

Según Gómez Orea (2007), se identifican 3 conceptos clave para la aplicación de la sostenibilidad en la acción y el cometido de las políticas de PT. En primer lugar la utilización racional del territorio y gestión responsable de los recursos naturales, implicando como principio general la conservación de los procesos ecológicos esenciales, de tal forma que se consiga mantener a largo plazo el potencial de utilización del suelo y los recursos que contiene. En segundo lugar la calidad ambiental, vinculada al objetivo de mejorar la calidad de los vectores ambientales (aire, agua y suelo), y la conservación de los ecosistemas y de los procesos ecológicos esenciales, del paisaje, del patrimonio cultural... etc. Finalmente, en tercer lugar, la calidad de la gestión pública y la coordinación administrativa, tomando como punto de partida la planificación integrada y la coordinación intersectorial e interterritorial entre los diferentes entes administrativos, destacando los que se deben producir entre entes del mismo nivel competencial (intercambio de información y cooperación activa). 


\section{La sostenibilidad en la planificación territorial}

El paradigma de la sostenibilidad está obligado a tener una nueva mirada sobre la realidad territorial. Se debe apostar por un nuevo concepto territorial, basado en la contención en el consumo de materiales y energía, en el vertido mesurado de residuos y el mantenimiento de la biodiversidad (Folch, 2003). El espacio libre es un bien, sobre todo cuando se vuelve escaso. Este bien ha sido excluido durante décadas de la ecuación territorial, lo que ha permitido su consumo sin freno. Un abordaje sistémico y sostenibilista del territorio, no puede admitir que el concepto espacio sea apartado de la hoja de cálculo a la hora de valorar situaciones territoriales. En general, no se puede admitir que se deseche de la imprescindible matriz biofísica.

Como nos indica Gómez Orea (2007, p. 101), tomando sus palabras textuales: "el DS pasa ineludiblemente por la consecución de un modelo territorial sostenible. La consecución de tal modelo es indisociable de la OT". La OT es una función que deben asumir las Administraciones Públicas, orientadas a conseguir el DS y equilibrado de la sociedad mediante la previsión de sistemas territoriales armónicos, funcionales y equilibrados capaces de proporcionar a la población una calidad de vida satisfactoria (Gómez Orea, 2007).

La implementación del proyecto territorial sostenibilista, debe centrar su atención en actuaciones de especial relevancia funcional, con el objetivo de maximizar la eficiencia de sus inversiones. Por eso, es conveniente poner el énfasis sobre los umbrales admisibles de impacto sobre el medio ambiente, más que sobre la disposición de las actividades en el espacio; sobre los ritmos y los procesos de transformación, más que sobre el tipo de transformación; sobre el control de las redes de interrelación, además del control de los elementos individuales; sobre diferentes escalas geográficas, ámbitos sectoriales y horizontes temporales; y sobre la participación de los agentes socioeconómicos y políticos que intervienen en los procesos de transformación del territorio (Folch, 2003).

Además, también aparecen prioridades espaciales, ligadas a los impactos que pueden derivarse a nivel territorial. Sus principales ideas a tener en cuenta son: la gestión socioambiental moderna y sostenible que se hace a una escala que sobrepasa la de los actuales municipios; el planeamiento que hay de tener en cuenta el heteromorfismo territorial y debe aplicarse sobre el espacio como una piel, más que como un corsé; el crecimiento, que no debe entenderse como un mero incremento del tamaño de las cosas; la estructura territorial, que debe permitir la gradual disminución de la movilidad obligada, confiándola más hacia los sistemas colectivos que los individuales; y el mantenimiento del espacio libre, normalmente como mejor opción para territorios de gran congestión (Folch, 2003).

Si se considera aquello que ha venido a denominarse como Nueva Cultura del Territorio (Tarroja, 2006), que hace referencia al desarrollo socio-ambiental del territorio, como una nueva aproximación de base territorial al concepto de DS, en la que se destacan las esferas sociales y ambientales, en un mayor grado que la esfera económica, existen siete conceptos básicos para el desarrollo socio-ambiental del territorio. 
Estos son los siguientes: 1) caminar hacia una estructura territorial articulada a través de una red policéntrica de sistemas urbanos que se reproduzca a distintas escalas; 2) articular un modelo urbanístico-territorial que evite la dispersión y la fragmentación urbana; 3) una nueva cultura de la movilidad; 4) una gestión prudente de los recursos ambientales, que reduzca el impacto ecológico y la gestión de la matriz de espacios abiertos y del paisaje; 5) la integración de las políticas de equidad y cohesión social en el modelo territorial; 6) la potenciación de los factores de competitividad del territorio, como son el capital humano, el social y el territorial; y 7) el establecimiento de redes de gobernabilidad de las políticas territoriales, basadas en la formulación participativa, el diálogo y la gestión concertada.

Por otra parte, si se consideran elementos más específicos de la PT, se puede indicar como por ejemplo Esteban (2006), destaca la necesidad de que los procesos de PT inicien dinámicas de contención de la dispersión urbana. En esta línea, resalta que el desarrollo urbanístico sostenible, dado que el suelo es un recurso limitado, comporta también la configuración de modelos que eviten la dispersión en el territorio. Las razones por las que la dispersión es un fenómeno negativo, se centran en: 1) el incremento del consumo de suelo; 2) la degradación del paisaje; 3) el impacto sobre los ecosistemas naturales; 4) la potencialización del uso del vehículo privado; y 5) el encarecimiento de la dotación y prestación de servicios. Resulta evidente pensar que la dispersión se produce porque existe una demanda para acceder a ese tipo de suelo más aislado o más periférico respecto de las zonas puramente urbanas (centros urbanos).
Esta realidad se genera por las características que ofrece este tipo de suelo, es decir, mayor calidad (tejidos de baja densidad, entornos espacialmente amplios, ajardinados, incluso con interés paisajístico), menor coste del suelo, exigencias dimensionales, posibilidades de implantación de instalaciones que requieran de amplias zonas, equipamientos deportivos, centros comerciales y recreativos, exigencias funcionales (alojamiento de actividades especialmente molestas o peligrosas), acciones de tipo cultural o de estatus social, mayores expectativas de nivel de vida, o de políticas de tipo fiscal.

Para poder evitar la demanda de suelo disperso no vale únicamente con establecer medidas restrictivas o limitativas en el planeamiento. Es necesario partir de un consenso social amplio, cosa que se puede conseguir cuando el planeamiento contribuya a facilitar otras opciones espaciales que pueden satisfacer algunos componentes de esa demanda. Entre estos, se puede destacar la calificación de suficiente cantidad de suelo de desarrollo urbano con buenas condiciones de conectividad con las áreas urbanas existentes, el asegurar buenos estándares de calidad ambiental (refuerzo de espacios con vegetación y establecimiento de densidades medias), la potenciación de la accesibilidad en transporte público para esos nuevos crecimientos urbanos, y el propiciar la construcción de viviendas, en especial asequibles, en las áreas donde haya o se prevea mayor proporción de puestos de trabajo, y viceversa (Esteban, 2006).

Desde este punto de vista de base territorial, como nos apuntan Elorrieta et al. (2016), la sostenibilidad está estrechamente relacionada con la capacidad de carga del 
propio territorio, y puede entenderse como "el desarrollo acorde con los rasgos ambientales del medio donde se plantean cambios en el uso del suelo. Esos rasgos ambientales incluyen cuestiones del medio físico, pero también aspectos socioeconómicos y culturales" (Elorrieta et al., 2016, p. 152).

Si se tienen en cuenta todas estas consideraciones planteadas en este apartado, el Plan se convierte un elemento fundamental para procesos importantes como; la participación y la concertación de los agentes socioeconómicos; la coordinación entre todos los agentes, de forma particular entre los numerosos organismos de la compartimentada administración pública; el establecimiento de la cultura de la previsión frente a la improvisación, en una forma de hacer operativo el principio relativo a la función social de la propiedad; y finalmente, en un objetivo que permite orientar los esfuerzos de todos en una misma dirección, cuyos resultados sólo se manifiestan y perciben a medio plazo (Gómez Orea, 2007).

En lo que respecta a la posición frente a las distintas modalidades de dispersión urbana, una de las cuestiones clave es conocer si a través de los planes urbanísticos municipales se puede actuar eficazmente 0 se necesitan instrumentos de mayor ámbito territorial. En relación con la dispersión y el crecimiento, además de las tres variables básicas, como son la localización, la morfología y la intensidad, resulta muy importante también identificar las necesidades de suelo y la aptitud de ese suelo en disposición de ser ocupado. Resulta bastante claro que las consideraciones sobre cuáles son los municipios con mejores condiciones, por su población, usos urbanos y conectividad, para dar respuesta a las necesidades de crecimiento, sólo pueden hacerse desde un enfoque territorial amplio. También es imprescindible este enfoque para valorar las mejores opciones de implantación urbana en función de las condiciones físicas del suelo disponible. El criterio de que cada plan municipal debe dar respuesta a la demanda interna de crecimiento del municipio, que se desprende de la legislación urbanística actual, únicamente tiene sentido en casos de municipios con disponibilidad de suelo y al margen de fenómenos metropolitanos. El grado de continuidad del crecimiento urbano con las áreas existentes y las densidades del mismo, sí son materia específica de los planes municipales, aunque dada la transcendencia territorial de estas decisiones cabe exigir criterios coherentes en todo el ámbito territorial significativo, los cuales sólo pueden ser aportados por un planeamiento con la suficiente amplitud (Esteban, 2006).

Desde una visión más práctica, si se toma en consideración las implicaciones de los principios de la sostenibilidad en la PT, según el Libro Blanco de la Sostenibilidad en el Planeamiento Urbanístico Español (2010), el planeamiento urbanístico define un modelo y una estructura de ciudad sobre la que posteriormente se instalan y desarrollan los distintos usos urbanos. En este modelo, aspectos, como las tipologías edificatorias y su relación con los espacios abiertos como viario, espacios de convivencia, zonas verdes, etc., la distribución de los distintos usos y su convivencia o separación de viviendas, equipamientos públicos y privados, usos terciarios e industriales). Así como su mayor o menor concentración en el espacio, pueden apoyar, e igualmente dificultar, determinados 
estilos de vida más o menos sostenibles. Por supuesto, en las sociedades democráticas la decisión última debería corresponder a los ciudadanos particulares, pero es labor de las administraciones públicas, tanto desde el planeamiento como desde otros ámbitos de su competencia, fomentar los hábitos individuales más beneficiosos para la colectividad, y que se ofrezcan los incentivos y desincentivos más apropiados para cada caso. Así pues, se ponen en juego una serie de elementos interrelacionados, como son: el territorio o soporte físico sobre el que funciona la ciudad, que ofrece un abanico de posibilidades de uso; la sociedad que hace un uso específico del soporte del que dispone, y que incluye su modificación; y el metabolismo urbano, con su correspondiente consumo de recursos y producción de residuos. Por tanto, el planeamiento urbanístico está encargado de conformar el soporte físico de la ciudad, pero al hacerlo influye necesariamente en las otras dos esferas. Desde la conciencia de esta capacidad de influencia pueden integrarse en el planeamiento estrategias de sostenibilidad.

\section{Criterios de aplicación práctica para la Planificación Territorial Sostenible}

Debido al modelo socioproductivo dominante, el único modo de avanzar hacia territorios más sostenibles que permitan alcanzar y mantener el bienestar de la población mundial, es tener presente la necesidad de planificar adecuadamente el desarrollo territorial. Como ya se ha indicado en el apartado introductorio del presente artículo, la ambigüedad que rodea al concepto del DS, complica en gran medida su aplicación práctica, en especial a la hora de implementarlo en las políticas de actuación territorial. Por este motivo, resulta fundamental avanzar en la definición de criterios para poner en marcha este tipo de políticas territoriales, tan necesarias para alcanzar los ya mencionados objetivos del DS, haciendo especial referencia a las escalas regionales y locales.

En los siguientes subapartados, tras una minuciosa revisión bibliográfica, y con el objetivo de aportar una base teórica amplia que facilite la aplicación práctica de la Planificación Territorial Sostenible, se definen de forma concreta los criterios que deben considerar. En el primero de estos subapartados, los criterios se describen desde un punto de vista general, mientras que en el segundo, se realiza una aproximación más específica, dividida por ámbitos funcionales de actuación, con una mirada más localista.

\section{Criterios generales}

El objetivo principal para que la PT contribuya a alcanzar el DS, tiene que pasar inevitablemente por construir un modelo territorial sostenible. Para poder alcanzar dicho modelo territorial, se debe partir de la consideración de una serie de principios o criterios generales, como elementos básicos para su consecución. A continuación se irán detallando aquellos que se consideran más importantes.

Una de las recomendaciones más relevantes para alcanzar la sostenibilidad es la de considerar el territorio como un elemento esencial para el desarrollo, teniendo 
que aceptar con ello su naturaleza sistémica y compleja (Folch, 2003). La políticas de PT deben fundamentarse en buenas políticas de gobernabilidad, basadas en la participación ciudadana, el diálogo y la gestión concertada (Tarroja, 2006).

La PT, como política de intervención planificada previsible, debe valorarse como la mejor opción ante la posible evolución espontánea (imprevisible) en la transformación del territorio. En este sentido se reforzarán los criterios de igualdad, proporcionalidad y de mejora de las condiciones de calidad de vida en general. (Pujadas y Font, 1998; Gómez Orea, 2007; Noguera et al., 2011). Al mismo tiempo, las políticas de PT deben aplicarse desde las diferentes escalas territoriales (nacionales, regionales y locales), mediante un desarrollo en cascada donde queden bien diferenciadas las estructuras correspondientes a cada nivel (Pujadas y Font, 1998; Gómez Orea, 2007; Noguera, 2009). También se debe partir de una concepción en la que se considere la necesaria integración de las políticas de PT con las políticas de equidad y cohesión social, con el objetivo de evitar la exclusión de sectores de la sociedad dentro del modelo territorial acordado (Tarroja, 2006).

La aplicación de la PT tiene que partir de la consideración inicial de los recursos endógenos de cada realidad territorial local, para que se promuevan actividades acordes con los problemas, aspiraciones, actitudes y aptitudes de su población (Gómez Orea, 2007). Los procesos de PT deben iniciarse desde la implicación de las comunidades locales, de modo que se movilice a sus agentes, y se facilite así el equilibrio territorial (Pujadas y Font, 1998; Benabent, 2006).
El heteromorfismo territorial, entendido como la gran variedad de realidades territoriales existentes en el planeta, necesita soluciones espaciales distintas, por lo que las políticas de PT deberán adaptarse a cada una de esas realidades y que se propicie la flexibilización en la aplicación de sus procesos (Folch, 2003). En esta línea, también se deberá ayudar a conseguir el equilibrio territorial, entendido éste como la igualdad de posibilidades entre territorios, con independencia de la escala territorial a la que se quiera hacer referencia (Carta Europea de la OT, 1983). Preservar los espacios naturales heredados (Pujadas y Font, 1998), y conservar el territorio en su estado original debe considerarse siempre como la primera opción, por lo que se tiene que justificar plenamente su necesidad de transformación. El espacio libre o espacio disponible es un bien a preservar (Pujadas y Font, 1998; Folch, 2003; Esteban, 2006; Farinós, 2006; Gómez Orea, 2007).

La utilización racional del territorio y practicar una gestión responsable de los recursos naturales en pro de la conservación de los procesos ecológicos (Folch, 2003), así como mejorar la calidad ambiental, a partir de tomar como base sus principales vectores (aire, agua y suelo) se consideran elementos fundamentales (Gómez Orea, 2001). Del mismo modo, es igualmente muy importante desarrollar una buena gestión pública y coordinación administrativa, traducidas éstas como el resultado de ejercicios de planificación integral y coordinación intersectorial e interterritorial (Gómez Orea, 2001; Farinós, 2006).

En los ámbitos más urbanos o metropolitanos, se tienen que tener en cuenta algunos principios más específicos. 
Este puede ser el caso de la asignación de usos del suelo concretos y diferenciados en función de las actividades desarrolladas por el hombre y sus necesidades (Pujadas y Font, 1998). La armonización de la ordenación de los diferentes usos del suelo, a partir de un enfoque territorial amplio, en el que la escala local encaje con el conjunto de usos establecidos en el área territorial superior en la que se integra (Esteban, 2006). Del mismo modo, se debe intentar evitar la dispersión y la fragmentación urbana (Tarroja, 2006; Esteban, 2006; Rojas et al., 2011), mejorar la calidad de los entornos urbanos existentes y de las nuevas zonas urbanas anexas a esos mismos entornos urbanos ya existentes, con el objetivo de equiparar sus condiciones de calidad, en comparación con las urbanizaciones aisladas (dispersas) y de baja densidad (Esteban, 2006), y, por último, disminuir la movilidad urbana obligada que esté necesariamente vinculada a la utilización de medios de trasporte particulares (Folch, 2003).

Finalmente, desde esta aproximación más general, destacar de nuevo la necesidad de alcanzar el consenso del conjunto de agentes sociales en la toma de decisiones, mediante la consideración como punto de partida de los procesos de participación ciudadana, en los que la población pueda expresar sus necesidades, siempre en beneficio del bien común (Gómez Orea, 2007; Libro Blanco de la Sostenibilidad de la Planificación Urbanística Española, 2010).

\section{Criterios específicos por ámbitos funcionales}

La puesta en práctica de los criterios generales indicados pasa por la definición de criterios específicos de aplicación. Este objetivo resulta fundamental para la aplicación sostenible de la PT. Para que dicha aproximación pueda ser entendida con mayor detalle, en este apartado los criterios se clasifican por ámbitos funcionales. Estos ámbitos son los que se consideran como los más relevantes para la implantación de actuaciones territoriales arraigadas en la escala local. A continuación, se van describiendo los criterios específicos que se consideran más importantes.

\section{Suelo urbano y suelo urbanizable}

Para poder hacer frente a la dispersión urbana y propiciar procesos de contención, resulta necesario evaluar las variables básicas asociadas a la planificación urbana. Estas son la localización, la morfología y la intensidad de las propuestas urbanas, las necesidades de suelo y la aptitud de los suelos (Esteban, 2006; Utz et al., 2008; Rojas et al., 2011). En relación con lo anterior, también se considera muy importante analizar las tipologías edificatorias propuestas y su relación con los espacios abiertos planificados (viarios, zonas verdes, corredores ecológicos) (OSE, 2009; Libro Blanco de la Sostenibilidad de la Planificación Urbanística Española, 2010). Por su parte, es igualmente necesario analizar la distribución (mezcla) de los usos del suelo, y su necesaria convivencia o separación, en función de sus características. Se considera como muy relevante la coexistencia espacial de usos, en especial de la mezcla de usos residenciales con usos funcionales o no residenciales útiles (servicios públicos, comercio y otros), con el objetivo de facilitar su integración espacial (Díaz et al., 2007; Utz et al., 2008; Salado et al., 2008; Libro Blanco de la Sostenibilidad de 
la Planificación Urbanística Española, 2010; Rojas et al., 2011).

Otro criterio fundamental, es el vinculado a la valoración del grado de concentración urbana propuesto, para garantizar un nivel mínimo de calidad ambiental urbana. Este elemento está directamente relacionado con la intensidad de las propuestas y la planificación de los espacios abiertos. Las densidades edificatorias son muy importantes, en el sentido que establecen los límites edificativos, y por tanto las capacidades máximas de albergar viviendas y en definitiva de población. En este sentido resulta también importante el grado de consolidación realmente aplicado, por cuanto marcará la densidad de ocupación o de habitantes por sectores o zonas. Los niveles de masificación de la población urbana guardan una relación directa con el grado de cumplimiento de los parámetros de calidad ambiental urbana ya mencionados (Parrado, 2001; Díaz et al., 2007; OSE, 2009; Libro Blanco de la Sostenibilidad de la Planificación Urbanística Española, 2010; Rojas et al., 2011).

La optimización del suelo urbano consolidado, la reutilización y renovación urbana, así como la recuperación de zonas degradadas y abandonadas, son otras claves importantes. En esta línea también resulta significativo conocer la proporción de espacios urbanos destinados a equipamientos y a zonas verdes urbanas (espacios abiertos) de estos espacios consolidados. Un criterio a seguir sería tener una zona verde accesible en un radio de 500 metros (Salado et al., 2008; Utz et al., 2008; Libro Blanco de la Sostenibilidad de la Planificación Urbanística Española, 2010). Dentro del ámbito de actuación de los espacios urbanos ya consolidados, la conservación del patrimonio cultural, entendido éste como los bienes de interés general que deben estar reconocidos con figuras de protección legal, es otra de las claves a tener en cuenta para una sociedad sostenible y con arraigo cultural (Utz et al., 2008; Salado et al., 2008).

En lo que respecta a los nuevos espacios propuestos para el crecimiento de los entornos urbanos, por las necesidades de la población, deben permitir un crecimiento permeable, que evite la formación de barreras y genere un sistema de espacios abiertos continuos (corredores verdes o infraestructura verde) (Díaz et al., 2007; y Utz et al., 2008; CITMA, 2012). Evitar la ocupación de los nuevos crecimientos en zonas consideradas de riesgo (naturales y posibles riesgos tecnológicos), así como respetar las tierras de uso agrícola y forestal de interés o gran fertilidad, a efectos de mantener los valores paisajísticos sin degradarlos y que se propicie espacios de transición entre el tejido urbano y los espacios rurales, son otros criterios significativos (Utz et al., 2008; Rojas et al., 2011).

\section{Suelo protegido}

En este ámbito, resulta importante mantener los niveles de protección y la superficie de los espacios que previamente ya contaban con algún nivel de protección espacial. De forma singular se deben respetar las afecciones identificadas en los estamentos territoriales superiores, ya sean desde las instancias Supranacionales (Unión Europea), de las nacionales o de las regionales (Pujadas y Font, 1998; Folch, 2003; OSG, 2007; Salado et al., 2008; Utz. et al., 2008; y OSE, 2009). Del mismo modo, intentar identificar espacios merecedores de protección de un interés especial en la escala 
local, tanto por su arraigo social y cultural, como por sus valores ecológicos, resulta fundamental. Atención especial merecen aquellos usos de carácter agrario o agroforestal, en los que la degradación de su mantenimiento y uso pudiera tener importante repercusiones ambientales (Gómez Orea, 2007; Rojas et al., 2011).

Otro criterio que se debe considerar en los suelos o espacios protegidos, son los relacionados con el mantenimiento y mejora o establecimiento de corredores ecológicos, que doten de sentido a la planificación territorial. También se deben incluir los espacios urbanos, para la creación de un sistema de espacios de abiertos infraestructura verde local y con una correcta integración en las escalas territoriales superiores (Rojas et al., 2011; CITMA, 2012).

\section{Ámbito social}

Evaluar los procesos de participación pública desarrollados, mediante la consideración de los ciudadanos como agentes que toman decisiones sobre el espacio en el viven y el tipo de entorno donde quieren vivir. Resulta interesante evaluar tanto la cantidad, como la calidad de los procesos realizados (Libro Blanco de la Sostenibilidad de la Planificación Urbanística Española, 2010; CITMA, 2012).

Si se entiende que la vivienda es un derecho de todos los ciudadanos, es necesario disponer de viviendas de carácter asequible para mejorar las condiciones de acceso al conjunto de la población. Se deben considerar tanto la cantidad de viviendas de protección oficial identificadas, como la localización de las mismas, con la idea de cumplir con los objetivos de integración social y reducción de la desigualdades sociales (Rojas et al., 2011; OSG, 2007; OSE, 2009).

\section{Calidad ambiental}

Se tiene que consideración de la calidad ambiental, en términos de calidad del aire, del agua y del suelo. En este sentido es importante conocer tanto la capacidad de suministro de los recursos hídricos necesarios para los usos planificados, como el sistema de gestión de aguas residuales resultantes del proceso de utilización (Gómez Orea, 2007; Rojas et al., 2011; CITMA, 2012). La calidad ambiental, tiene igualmente una relación directa con el grado contaminación acústica de los espacios del territorio que sufren un alto grado de saturación. (Gómez Orea, 2007; CITMA, 2012).

La gestión de los vertidos o desechos urbanos es también una pieza clave. Es necesario identificar espacios para el tránsito y clasificación de los desechos. Estos sistemas requieren de una integración territorial superior, especialmente en los niveles comarcales/ regionales, que se estiman como los más apropiados para estos menesteres, aunque no deben quedar desconectados de sus sistemas territoriales superiores (Folch, 2003; Libro Blanco de la Sostenibilidad de la Planificación Urbanística Española, 2010; CITMA, 2012).

\section{Movilidad sostenible e infraestructuras de transporte}

En este ámbito funcional, uno los elementos más importantes a considerar, es el que tiene que ver con la identificación de las necesidades de movilidad diaria de la población, vinculadas a los desplazamientos a los lugares de trabajo o de estudio, y en función del colectivo social al que se pertenezcan. (Parrado, 2001; Díaz et al., 2007; OSG, 2007; Libro Blanco de la Sostenibilidad de la Planificación Urbanística Española, 2010). Resulta también, muy 
importante analizar los parámetros de accesibilidad considerados, que deben primar la implantación de sistemas de transporte públicos, a partir de una perspectiva territorial de amplitud de miras, con la integración de los servicios en las escalas supralocales (Libro Blanco de la Sostenibilidad de la Planificación Urbanística Española, 2010; y Rojas et al., 2011). Esta circunstancia lleva a la necesaria integración de la movilidad y el transporte público en la planificación de los usos de suelo finalmente propuestos (Rojas et al., 2011).

Para poder aplicar correctamente los criterios de movilidad sostenible definidos, es crucial contar con un buen conocimiento de la distribución espacial de la red de carreteras, la superficie ocupada y de su predisposición a ejercer de barreras ecológicas y ciudadanas. (Díaz et al., 2007; OSE, 2009; Rojas et al., 2011). Por último, también resulta fundamental evaluar la distribución de los espacios especiales para la práctica del desplazamiento - la movilidad personal sostenible. Cabe destacar el papel de los carriles de bicicletas y de peatones, con una clara diferenciación por uso, para evitar accidentes.(OSG, 2007; OSE, 2009; CITMA, 2012).

\section{Conclusiones}

Como se ha podido observa a lo largo del presente artículo, la Planificación Territorial Sostenible resulta crucial para que nuestras sociedades afronten el reto del DS, y de forma especial desde su interpretación en las escalas regionales y locales. El territorio es un factor vital a considerar para propiciar políticas de desarrollo, no incorporar esta matriz de carácter biofísico en las fórmulas del desarrollo, sería un error de gran magnitud y de difícil resarcimiento.

En línea con este planteamiento, y como conclusión, resulta importante identificar como protagonista principal de los procesos de Planificación Territorial Sostenible, al mencionado factor territorio, reconociendo su funcionamiento como un sistema, y en el que el espacio libre debe ser un bien a preservar. Así mismo, se tiene que destacar igualmente la necesidad de apostar por modelos de ciudades compactas, evitando la fragmentación y la dispersión urbana como factores clave para mejorar los grados de sostenibilidad. En estos espacios urbanos, resulta igualmente importante posibilitar la mezcla de usos residenciales con usos funcionales o no residenciales útiles (servicios públicos, comercio y otros), con el objetivo de facilitar su integración espacial.

Todas estas pautas o criterios, contribuirán a la disminución de la movilidad urbana obligada, otro de los condicionantes más importantes en los parámetros de insostenibilidad de las ciudades. Además, también es crucial primar la permeabilidad territorial para un mejor funcionamiento del ecosistema natural y ofrecer una función más sostenible a los espacios urbanos, mediante la generación de sistemas de espacios abiertos que actúen de corredores verdes urbanos. Finalmente, hay que destacar la importancia de los procesos de gobernanza territorial y la aplicación de procesos de participación ciudadana para mejorar la concertación de las actuaciones y facilitar la participación pública. 


\section{Adrián Ferrandis Martínez}

Universitat de València, Instituto Interuniversitario de Desarrollo Local, Departamento de Geografía. Valencia, Espanha.

adrian.ferrandis@uv.es

\section{Joan Noguera Tur}

Universitat de València, Instituto Interuniversitario de Desarrollo Local, Departamento de Geografía. Valencia, Espanha.

joan.noguera@uv.es

\section{Nota}

(1) En realidad la mayoría de estos nuevos planteamientos parte de viejas propuestas que han sido retomadas y reformuladas, como las teorías clásicas del estado estacionario o crecimiento cero, entre otras.

\section{Referencias}

BENABENT FERNÁNDEZ DE CÓRDOBA, M. (2006). La ordenación del territorio en España; evolución del concepto y de su práctica en el siglo XX. Colección Kora n. 16. Universidad de Sevilla y la Consejería de Obras Públicas y Transportes de la Junta de Andalucía. Sevilla.

BESSET, J.P. (2005). Comment ne plus être progressiste... sans devenir reáctonnaire. Paris, Fayard.

BRUNDTLAND, H. G. (1987). Nuestro Futuro Común, Comisión mundial del medio ambiente y del desarrollo. Naciones Unidas.

CITMA - Conselleria d'Infraestructures, Territori y Medi Ambient de la Generalitat Valenciana (2012). Documento de Referencia informado positivo correspondiente al Plan General de Pedreguer (Alicante), para la posterior elaboración del Informe de Sostenibilidad Ambiental.

CONSEJO DE EUROPA (1983). Carta Europea de Ordenación del Territorio. Conferencia europea de ministros responsables de la ordenación del territorio. Torremolinos, España.

CRUZ VILLALÓN, J. (2006). "Prologo", en La ordenación del territorio en España; evolución del concepto y de su práctica en el siglo XX. Colección Kora n. 16. Universidad de Sevilla y la Consejería de Obras Públicas y Transportes de la Junta de Andalucía. Sevilla.

DÍAZ MUÑOZ, M. A.; CANTERGIANI, C. C.; SALADO GARCÍA, M. J.; ROJAS QUEZADA, C. y GUTIÉRREZ MARTÍNEZ, S. (2007). Propuesta de un sistema de indicadores de sostenibilidad para la movilidad y el transporte urbanos. Aplicación mediante SIG, a la ciudad de Alcalá de Henares. Cuadernos de Geografía, v. 81-82, pp. 31-50. 
DOUGLAS MUSCHETT, F.; LEE CAMPBELL, C.; EVANS, V.; HENCK, W.W.; SI DUK LEE, V. S.; SHEN, T. y WARRER, J. L. (1999). Principios del Desarrollo Sostenible. Madrid, Aenor.

DOUGLAS MUSCHETT, F. (1999). Un método Global para el desarrollo sostenible. Principios del Desarrollo Sostenible. Madrid, Aenor, pp. 17-63.

ELORRIETA, B.; OLCINA, J. y SÁNCHEZ, D. (2016). La sostenibilidad en la planificación territorial de escala regional. Cuadernos Geográficos, v. 55, n. 1, pp. 149-175.

ESTEBAN, J. (2006). La necesaria contención de la dispersión urbana. Nueva cultura del territorio. Criterios sociales y ambientales en las políticas y el gobierno del territorio. Colección Territorio y Gobierno: Visiones, v. 4. Diputación de Barcelona. Barcelona, pp. 267-277.

FARINÓS DASÍ, J. (2006). La Estrategia Territorial Europea en el nuevo paradigma de la territorialidad. Nueva cultura del territorio. Criterios sociales y ambientales en las políticas y el gobierno del territorio. Colección Territorio y Gobierno: Visiones, v. 4. Diputación de Barcelona. Barcelona, pp. 43-68.

FERRANDIS MARTÍNEZ, A. (2016). A propósito del Desarrollo Sostenible: origen, evolución y teorías alternativa. Terra revista de desarrollo local, n. 2. Instituto Interuniversitario de Desarrollo Local, Universitat de València, pp. 74-96.

FOLCH, R. (coord.) (2003). El territorio como sistema. Conceptos y herramientas de ordenación. Colección Territorio y Gobierno: Visiones, v. 3. Diputación de Barcelona. Barcelona.

GÓMEZ OREA, D. (2001). Ordenación territorial. Coedición Mundi-Prensa y Editorial Agrícola Española. Madrid.

(2007). Evaluación Ambiental Estratégica: un instrumento para integrar el medio ambiente en la elaboración de planes y programas. Madrid, Mundi-Prensa.

GOODLAND, R. (1997). Introducción, en Medio ambiente y desarrollo sostenible. Más allá del Informe Brundtland. Colección de Estructuras y Procesos. Serie de Medio Ambiente. Editorial Trotta. Madrid. Traducción de Environmentally sustainable economic development: Building on Brundtland.

GOODLAND, R.; DALY, H.; EL SERAFY, S. y VON DROSTE, B. (eds.) (1997). Medio ambiente y desarrollo sostenible. Más allá del Informe Brundtland. Colección de Estructuras y Procesos. Serie de Medio Ambiente. Editorial Trotta. Madrid. Traducción de Environmentally sustainable economic development: Building on Brundtland.

LATOUCHE, S. (2008). La apuesta por el decrecimiento: ¿Cómo salir del imaginario dominante? Colección de ecología Antrazyt, n. 273. Capellades (Barcelona), Editorial Icaria.

LEE CAMBELL, C. y HECK, W. W. (1999). La perspectiva ecológica del desarrollo sostenible. Principios del Desarrollo Sostenible, pp. 65-87, Madrid, Aenor.

MINISTERIO DE LA VIVIENDA; GOBIERNO DE ESPAÑA (2010). Libro Blanco de la Sostenibilidad en el Planeamiento Urbanístico Español. Dirección Fariña Tojo, J. y Naredo, J. M. Madrid.

NAREDO, J. M (1996). Sobre el origen, el uso y el contenido del término sostenible. Primer catálogo español de buenas prácticas, pp. 21-28. Madrid, Ministerio de Fomento. 
NOGUERA TUR, J. (2009). Pensamiento y planificación estratégica: definición e implementación de estrategias de desarrollo. Gestión y Promoción del Desarrollo Local, serie de Estudios y Documentos de la colección Desarrollo Territorial, v. 4. Instituto Interuniversitario de Desarrollo Local, pp. 57-84, serie de Publicaciones de la Universitat de València.

NOGUERA TUR, J.; FERRANDIS MARTÍNEZ, A.; RIERA SPIEGELHALDER, M. y MORANT ESPARCIA, M. (2011). Análisis del impacto del proyecto "Vall de la Innovació" y de su compatibilidad con los objetivos y principios directores de la Estrategia Territorial de la Comunitat Valenciana. Instituto Interuniversitario de Desarrollo local de la Universitat de València.

OBSERVATORIO DE LA SOSTENIBILIDAD DE GUIPÚZCOA (2007). Sistema de Indicadores de Sostenibilidad de Guipúzcoa. Diputación Foral de Guipúzcoa, Departamento para el Desarrollo Sostenible, Dirección General de Medio Ambiente.

OBSERVATORIO DE LA SOSTENIBILIDAD EN ESPAÑA (2009). Sostenibilidad Local: una aproximación urbana y rural. Informes temáticos. Ministerio de Medio Ambiente de España, la fundación de la Universidad de Alcalá y la Fundación Biodiversidad.

PARRADO DELGADO, C. C. (2001). Metodología para la ordenación del territorio bajo el prisma de la sostenibilidad (estudio de su aplicación en la ciudad de Bogotá). Universitat Politècnica de Catalunya, Departament de d’Enginyeria Minera y Recursos Naturals.

PUJADAS, R. y FONT, J. (1998). Ordenación y Planificación Territorial. Ed. Síntesis, Colección Espacios y Sociedades, Madrid.

RAE - Real Academia Española (2014). Diccionario de la lengua española. Asociación de Academias de la Lengua Española.

RAMONET, I. (2003). Salvar el planeta. Salvar el planeta; ecología y desarrollo sustentable. Selección de artículos publicados en Chile. Editorial Aún Creemos en los Sueños. Santiago de Chile.

REDCLIFT, M. (2000). El desarrollo sostenible; necesidades, valores, derechos. Desarrollo sostenible; un concepto polémico. Serie de Ciencias Sociales. Universidad del País Vasco, Bilbao. Volumen n. 8, pp. 17-38.

ROJAS, C.; SALADO, M. J.; PINO, J.y MARTORI, J. C. (2011). Medidas para la valoración de la sostenibilidad de los territorios metropolitanos: aplicación al área metropolitana de la Concepción (Chile). Boletín de la Asociación de Geógrafos Españoles, n. 55, pp. 81-99.

RUEDA, S.; DE CÁCERES, R.; CUCHÍ, A. y BRAU, L. I. (2012). El Urbanismo Ecológico; su aplicación en el diseño de un ecobarrio en Figueres. Agència d’Ecologia Urbana de Barcelona.

SALADO GARCÍA, M. J.; ROJAS QUEZADA, C. y CARVALHO CANTERGIANI, C. (2008). Modelos Territoriales y Sistemas de Indicadores de Sostenibilidad. Valoración Crítica". Serie de Geografía - Profesora María de los Ángeles Díaz. In Memorian, n. 14, pp.131-145.

SHEN, T. (1999). La importancia del papel de la tecnología en la consecución del desarrollo sostenible. Principios del Desarrollo Sostenible, pp. 88-119. Madrid, Aenor.

TARROJA, A. y CAMAGNI, R. (coord.) (2006). Nueva cultura del territorio. Criterios sociales y ambientales en las políticas y el gobierno del territorio. Colección Territorio y Gobierno: Visiones, v. 4. Diputación de Barcelona. Barcelona.

URTEAGA, E. (2011). Las teorías alternativas del desarrollo sostenible. Boletín de la Asociación de Geógrafos Españoles, n. 55, pp. 113-126. 
UTZ, R.; JAQUE, E.; ROJAS, C. y SUAZO, B. (2008). Evaluación de sostenibilidad de la planificación territorial de los espacio urbanos costeros: caso de estudio. Ciudad de Penco. Chile.

VILCHES, A. y GIL, D. (2003). Construyamos un futuro sostenible; Diálogos de supervivencia. Colección Ciencia, Tecnología, Sociedad e Innovación, Cambridge University Press en colaboración con la Organización de Estados Iberoamericanos, Madrid.

VON DROSTE, B. y DOGSÉ, P. (1997). El desarrollo Sostenible. El papel de la inversión. Medio ambiente y desarrollo sostenible. Más allá del Informe Brundtland. Colección de Estructuras y Procesos, Serie de Medio Ambiente, pp. 87-99. Madrid, Editorial Trotta.

Texto recebido em 29/abr/2016

Texto aprovado em 21/ago/2016 
